\title{
Comparative Analyses of Chloroplast Genomes in 'Red Fuji' apples: Low rate of chloroplast genome mutations
}

\author{
Haoyu Miao ${ }^{\text {Equal first author, } 1}$, Jinbo Bao ${ }^{\text {Equal first author, } 1}$, Xueli Li ${ }^{1}$, Zhijie Ding $^{1}$, Xinmin Tian $^{\text {Corresp. } 1}$ \\ ${ }^{1}$ Xinjiang Key Laboratory of Biological Resources and Genetic Engineering, College of Life science and Technology, Xinjiang University, Xinjiang, Urumqi, \\ China \\ Corresponding Author: Xinmin Tian \\ Email address: tianxm06@Izu.edu.cn
}

Background. Fuji is a vital apple cultivar, and has been propagated clonally for nearly a century. The chloroplast genome variation of Fuji apples in China has not been investigated. Methods. This study used next-generation high-throughput sequencing and bioinformatics to compare and analyze the chloroplast genome of 24 Red Fuji varieties from nine regions in China. Results. The results showed that the 24 chloroplast genomes were highly conserved in genome size, structure, and organization. The length of the genomes ranged from 160,063 to $160,070 \mathrm{bp}$, and the GC content was $36.6 \%$. Each of the 24 chloroplast genomes encoded 131 genes, including 84 protein-coding genes, 37 tRNA genes, and eight rRNA genes. The results of repeat sequence detection were consistent; the most common sequence was forward repeats (53.1\%), and the least common sequence was complementary repeats (4.1\%). The chloroplast genome sequence of Red Fuji was highly conserved. Two indels were detected, but the PI value was 0 , and there were no SNP loci. The chloroplast genome variation rate of Red Fuji was low. 
1

2 Comparative Analyses of Chloroplast Genomes in

3 'Red Fuji' apples: Low rate of chloroplast genome

4 mutations

5

6

7

8

9

${ }^{1}$ Xinjiang Key Laboratory of Biological Resources and Genetic Engineering, College of Life 10

11

12

13

14 Corresponding Author:

15 Xinmin $\operatorname{Tian}^{1}$

16 Shengli Road 666, Urumqi 830046, Xinjiang, China

17 Email address: tianxm06@1zu.edu.cn

18 
19

20

21

22

23

24

25

26

27

28

29

30

31

32

33

34

35

36

37

38

39

40

41

42

43

44

45

46

47

48

49

\section{Abstract}

Background. Fuji is a vital apple cultivar, and has been propagated clonally for nearly a century. The chloroplast genome variation of Fuji apples in China has not been investigated.

Methods. This study used next-generation high-throughput sequencing and bioinformatics to compare and analyze the chloroplast genome of 24 Red Fuji varieties from nine regions in China. Results. The results showed that the 24 chloroplast genomes were highly conserved in genome size, structure, and organization. The length of the genomes ranged from 160,063 to 160,070 bp, and the GC content was 36.6\%. Each of the 24 chloroplast genomes encoded 131 genes, including 84 protein-coding genes, 37 tRNA genes, and eight rRNA genes. The results of repeat sequence detection were consistent; the most common sequence was forward repeats $(53.1 \%)$, and the least common sequence was complementary repeats (4.1\%). The chloroplast genome sequence of Red Fuji was highly conserved. Two indels were detected, but the PI value was 0 , and there were no SNP loci. The chloroplast genome variation rate of Red Fuji was low.

\section{Introduction}

Apple (Malus $\times$ domestica) belongs to the genus Malus in the family Rosaceae. It is among the most popular and nutritious fruits (Jung \& Main, 2014), In 2018, China, which is the largest producer of apples in the world (Li et al., 2019), produced more than half (43.88 million tons) of the global production (80.82 million tons). In addition to being used as fresh, natural products, apples can also be processed into foods, like apple juice, applesauce, and dried fruits (Deng \& $L i$, 2020). Apple cultivation has a significant value and significance in China's economic development. Many excellent varieties, such as 'Fuji' have been widely cultivated (Kuang et al., 2020). 'Fuji' was generated in Japan from 'Ralls Janet' $\times$ 'Delicious '(Hummer \& Janick, 2009). 'Fuji' apple is one of China's most popular late-ripening varieties, accounting for $72.7 \%$ of the total Chinese apple production (An et al., 2009). Fuji has many advantages, such as colorfulness, sweet taste, storage resistance, and high profit. It is sweeter and crisper than many other apple varieties, and has a longer shelf-life (Li et al., 2019). It is widely consumed worldwide due to its excellent properties (He \& Zhang, 2020). The economic value of 'Fuji' apples, highlights the importance of research on breeding and crop improvement. Most of the research on Fuji apples has concentrated on the nuclear genome. Research on organellar genomes is scarce. The nonnuclear genome includes the chloroplast (cp) and the mitochondrial genomes. 
50

51

52

53

54

55

56

57

58

59

60

61

62

63

64

65

66

67

68

69

70

71

72

73

74

75

76

77

78

79 80

81

80

Chloroplasts have an independent genetic system and are semi-autonomous organelles (Sugiura, 2003). In 1978, the first chloroplast gene was isolated using molecular cloning by Rochaix et al. (1978). Some years later, Ohyama et al. (1986) and Shinozaki et al. (1986) sequenced the whole $\mathrm{cp}$ genome of Nicotiana tabacum and Marchantia polymorpha. For several reasons, the cp genome is more commonly used in molecular evolution and phylogenetic studies than the mitochondrial genome (Guo et al., 2021). Firstly, the cp genome includes a large amount of genetic information, which provides a database for comparative research (Zuo et al., 2017). Secondly, the nucleotide substitution rate is moderate and valuable in evolutionary studies (Moore et al., 2010). The molecular evolutionary rates in various regions of the $\mathrm{cp}$ genome are significantly different and could be used for different levels of systematic research ( Li et al., 2018). In addition, the moderate cp genome size is easy to sequence, and there is good collinearity between the cp genomes of various plant groups, making it convenient for comparative analysis (Alzahrani, 2021). Therefore, the cp genome has a significant impact on the development of phylogenetic genomics (Wang et al., 2021). The number of cp genomes that have been sequenced has increased exponentially owing to the development of sequencing technology (Liu et al., 2012) The cp genome has been successfully used to analyze the phylogenetic relationships between numerous difficult species and study the structural characteristics, variation, and evolution of plants (Yagi \& Shiina, 2014). For example, Jansen (2007) used $64 \mathrm{cp}$ genomes to determine the phylogenetic relationship between angiosperms. Moreover, through homology analysis of the cp genome, we can deal with crop origin and domestication's important scientific problems and detect the changes in crop genome structure and gene sequence during domestication (Abdullah et al., 2020).

Yan et al. (2019) assembled and annotated the cp genome of cultivated apple (Yantai Fuji 8), which enriched the potential genetic resources for apple breeding. The quality of the same apple species can vary greatly in different plantations because of climate (Fu et al., 2013). Genetic changes of the genome can occur rapidly (Jiao et al., 2014). After nearly a century of clonal propagation, it is unknown whether the cp DNA of Red Fuji apples planted in different areas differs and whether there is a variation. This study, characterized the complete cp genome sequence of 24 Red Fuji accessions in nine regions and carried out a structural variation analysis. This study aimed to investigate mutations in the chloroplast DNA of the Red Fuji apple.

\section{Materials \& Methods}


82

83

84

85

86

87

88

89

90

91

92 93

97

98

\section{Plant materials}

Germplasm of 24 Red Fuji apples was collected from nine regions in China. Two or three accessions were selected from each region for repeated experiments. The sources and quantities of the accessions are listed in Table 1. Fresh leaves of Red Fuji were dried in silica gel and taken to the laboratory.

\section{DNA extraction and sequencing}

Total genomic DNA of each material was extracted using the plant genomic DNA secure Kit (DP320) of China Tiangen Biotechnology (Beijing) Co., Ltd. The integrity and purity of the DNA samples were determined by agarose gel electrophoresis. NuoheZhiyuan Technology Co., Ltd. (Beijing, China) used the Illumina Hiseq 2000 sequencing platform to perform genome sequencing of quality DNA samples. The read length was $150 \mathrm{bp}$. A total of $492.617 \mathrm{G}$ of raw data was generated by sequencing, and the filtered clean data was $491.884 \mathrm{G}$, with an average of 20987.043M per sample (Table S1).

\section{Plastome assembly and annotation}

We chose a reference map for assembly. The obtained clean data were assembled using Novoplasty software (https://github.com/ndierckx/ NOVOPlasty), the configuration file config.txt needs to be set before use. Download the published chloroplast genome of Malus yunnanensis (GB: MH394390) from NCBI as the reference file, select its $r b c$ Lene as the seed file, $\mathrm{kmer}=39$. The preliminary annotation of the assembled plastome was performed using Geseq (https://chlorobox.mpimp-golm.mpg.de/geseq.html). Then, Sequin 16.0 was used to correct the start and stop codons and intron/exon boundaries for the genes with software annotation errors. Finally, the complete cp genome sequence and its annotations were submitted to GenBank. The specific serial numbers are detailed in Table 1.

\section{Characterization and comparative analyses of chloroplast genomes}

The total length of the genome, the length of each region (large single-copy regions, small single-copy regions, inverted repeat), gene composition, base composition and GC (AT) content were calculated using Geneious 11.1.2 software, The characteristics of Red Fuji genomes were analyzed.

\section{Boundary regions and comparative analysis}


116 Different plant species have different gene sequences in the four junction regions. The change of

117 cp genome length is the main reason for the contraction and expansion of the IR region

118 (Raubeson et al., 2007). The border regions between large single copy (LSC) and IR regions, and

119 between small single copy (SSC) and IR regions, were compared using IRscope

120 (https://irscope.shinyapps.io/irapp/).

121

122 Codon usage analyses

123 Codon usage bias (CUB) refers to the phenomenon that some codons are used more than other 124 synonymous codons in the process of gene translation between different species or within the 125 same species (Alexandra \& Tamir, 2014). CUB is a useful tool for understanding genetic and 126 evolutionary processes. In this study, MEGA7.0 software CodonW was used to analyze codon 127 preference, The results of the analysis are shown in charts.

128

129

130

\section{Repeat sequence and SSRs analyses}

The online REPuters (https://bibiserv.cebitec.unibielefeld.de/repuer) software was used to identify repeats which contain forward, reverse, and complex, repeats as well as palindromes of the cp genome of Red Fuji (Frazer et al., 2001). The following parameters were used to identify repeats with REPuter: Hamming distance of 3, Maximum Computed Repeats of 50, and repeat size $>30 \mathrm{bp}$. Simple sequence repeats (SSRs) were checked using MISA (https://webblast.ipkgatersleben.de/misa/index.php), with motif sizes of 1-6 nucleotides (Thiel et al., 2003). All other parameters were used as default values. The parameters of repetitive units and the minimum number of repetitions were set to 10 repetitions for single nucleotide type, 6 repetitions for dinucleotide type, 5 repetitions for trinucleotide type, 5 repetitions for tetranucleotide type, 5 repetitions for pentanucleotide type, and 5 repetitions for hexanucleotide type.

140

\section{Genome comparison}

142 The cp genomes of Red Fuji apples from nine regions were compared and visualized by

143 mVISTA (http://genome.lbl.gov/vista/index.shtml) online software . mVISTA is an online tool

144 used for multiple DNA sequence alignments, where sequence similarity can be evaluated by 145 comparing coding regions with non-coding regions, introns, and exons (Frazer et al., 2004). The 146 genome of $M$. sieversii was selected as the reference, and the input file was the original FASTA 147 format nucleotide sequence file and the gff3 format annotation file. The nucleotide diversity (PI) 
148 of Red Fuji was calculated in Dnaspv6 software. SNP and indel detection was carried out using

149 Geneious 11.1.2

150

151 Phylogenetic analysis

152 A phylogenetic tree was constructed based on the combined cp genomes. Pyrus pyrifolia was

153 used as the outgroup. The species and the accession numbers of their cp genomes in NCBI are

154 listed in Table S2. The sequences were aligned using Mafft

155 (https://mafft.cbrc.jp/alignment/server/). The model is GTRGAMMA+I. Maximum Likelihood

156 (ML) methods were used to construct phylogenetic trees was used to perform the RAxML-HPC

157 BlackBox 8.2.12 in CIPIES (https://www.phylo.org/) (Stamatakis, 2014) the ML analysis

158

159 Results

160 Characteristics of Red Fuji cp genomes

161 The $24 \mathrm{cp}$ genomes of the Red Fuji apples collected were highly conserved in terms of gene

162 content, gene order, and gene intron number. The genomes ranged in length from 160,063 bp to

$163160,070 \mathrm{bp}$, and as expected, the cp genomes contained the LSC and SSC, separated by a pair of

164 IR regions (Fig. 1). The length of the IR region was 26,307 - 26,308 bp, the LSC region was

16588,272 - 88,274bp, and the SSC region was 19,176 - 19,181 bp. The overall GC content was

166 approximately $36.6 \%$. The GC content in the IR region was $42.7 \%$, which was higher than

$16734.2 \%$ in the LSC region and $30.4 \%$ in the SSC region (Tables2 and 3). These numerical values

168 were consistent among the 24 accessions and did not change.

169 Each plastome encoded 131 genes, including 84 protein-coding genes (PCGs), 37 transfer

170 RNA (tRNA) genes, and eight ribosomal RNA (rRNA) genes. There were 22 introns in the

171 annotated genes. Of the annotated genes, 18 embodied one intron, and two ( $y c f 3$ and $\operatorname{clpP}$ ) had

172 two introns. Additionally, 62 protein-coding and 22 tRNA genes were located within the LSC;

17312 protein-coding genes, 14 tRNA coding genes, and 8 rRNA coding genes were located within

174 IRs, and 12 protein-coding genes and one tRNA gene were located within SSC. The genes in the

175 LSC region accounted for $64.1 \%$ of the cp genome, the two IR regions accounted for $26 \%$, and

176 the genes in the SSC region accounted for $9.9 \%$.

177

178 IR boundary analysis

179 First, IR analysis was performed on the materials from each region, and the results showed that

180 the cp genomes of Red Fuji in the same region were highly consistent (Fig. S1-S9). Therefore, 
181 the subsequent analysis was based on comparing the nine regions Nine samples were selected

182 from each region to compare the distribution of genes in the boundaries of the four regions of the 183 cp genome. The IR regions of the nine Fuji genomes sequenced were highly conserved (Fig.

184 S10). We compared gene variation at the boundaries of five plastosomes of three Red Fuji, one 185 M. sieversii (MK434920), and one M. sylvestris (MK434924). The results displayed that the 186 boundaries of the four regions were relatively conserved, and the gene types distributed in the 187 boundary region were highly consistent. The genes distributed in the boundary between the LSC 188 and IR region were $r p s 19$ and trnH-GUG. In addition, $r p 12, y c f 1$, and $n d h \mathrm{~F}$ are distributed in the 189 boundary of the SSC / IR region. (Fig. 2). The length of $y c f 1$ extending to the IR region was $1901,074 \mathrm{bp}$, and that of $n d h \mathrm{~F}$ was $12 \mathrm{bp}$. The length of rps 19 extending to the IRb region extended 191 was 69 or $115 \mathrm{bp}$, depending on the different species. The position of the gene in the boundary 192 region was relatively fixed. There were no differences in the boundaries of the five samples. 193 Hence, these results indicate that the IR region is highly conserved

194

195 Codon usage analyses

196 Since the base composition and AT/GC content was the same in the 24 genomes of Red Fuji, we 197 chose one of them to calculate the frequency of amino acid and codon usage. The codon usage 198 results of materials from Wunan Town, Wuwei City showed that a total of 20 amino acids 199 (excluding the stop codon) were encoded, and the usage frequency of each amino acid ranged 200 from $1.56 \%$ to $9.38 \%$. Leucine, serine, and arginine were the most abundant amino acids. 201 Among the encoded amino acids, except methionine and tryptophan, other amino acids were 202 encoded by two to six synonymous codons (Fig. 3). For example, serine, arginine, and leucine 203 were encoded by six synonymous codons, of which the most frequently used codons were TTA, 204 TCT, and CGT, respectively. Four synonymous codons were used for valine, proline, threonine, 205 alanine, and glycine. The most frequently used codons were GTT, CCT, ACT, GCT, and GGT, 206 respectively. Three synonymous codons were used for isoleucine, of which ATT was the most 207 frequently used codon. Two synonymous codons were used for phenylalanine, tyrosine, 208 histidine, glutamine, asparagine, lysine, aspartic acid, glutamic acid, and cysteine, which had 209 different frequencies of use. The codons with a higher frequency for these nine amino acids were 210 TTT, TAT, CAT, CAA, AAT, AAA, GAT, GAA, and TGT, respectively. The cp genome of Red 211 Fuji apple has 32 codons with RSCU $>1$, of which 30 codons end with A/T, accounting for $21293.75 \%$. Most of the 29 codons with relative synonymous codon usage values (RSCU) 1 ended 
213 in $\mathrm{G} / \mathrm{C}$, accounting for $96.55 \%$. Our results were similar to those of other angiosperms. The

214 codons of the cp genome of Red Fuji that were used frequently at the third codon positions were

215 A and $\mathrm{T}$.

216

217 Repeats and microsatellites analyses

218 A total of 49 repeat sequences were detected in each material, which consisted of 26 forward, 17 219 palindromic, 4 reverse, and 2 complement repeats(Table S3). Among them, there are 33 repeats 220 with a length of 30-39 bp, 6 repeats with a length of 40-49 bp, and two repeats with more than 50 221 bp (Fig. 4). The most common type was forward repeats (53.1\%), and the least common was 222 complement-type repeats (4.1\%). Using the MISA software, we searched for the SSR loci in the 22324 Red Fuji apple cp genomes. A total of 1,380 microsatellites, two dinucleotide repeats, 46 to 22448 mononucleotides repeats, and 8 or 9 complex polynucleotides were found. Mononucleotides 225 (81.2\%), mainly poly-A (polyadenine) and poly-T (polythymine), formed the largest proportion 226 of SSRs in the Red Fuji cp genome. The majority of SSRs were situated in the intergenic region 227 of LSC (Table.4).

228

\section{Genome comparison and divergence analyses}

230 In this study, the online software mVISTA was used for sequence alignment and variation 231 analysis of cp genome of Red Fuji, using M. sieversii (MK434920) as the reference. The 232 nucleotide sequence similarity of the ten cp genomes was extremely high, suggesting that there 233 was no variation in the cp genome of Red Fuji compared with its ancestral species (Fig. 5). At 234 the same time, it can be found that divergence existed in the highly conserve dregions, and the 235 coding region and IR region were more conserved. The intergenic regions with the highest levels 236 of divergence were those in between $p s b \mathrm{~L}-a t p \mathrm{~A}, p s b \mathrm{M}-p s b \mathrm{D}$, and $n d h c-a t p \mathrm{E}$.

237 The nucleotide polymorphisms were analyzed using the DNAsp software, and the nucleotide 238 diversity (PI) was 0 . Geneious was used to detect nine regions' single nucleotide polymorphisms 239 (SNPs) of M. sieversii, M. sylvestris, and Red Fuji apples in nine regions, Only two indel loci 240 were detected in the $r p s 16$ and $n d h \mathrm{~A}$ genes. The results suggested that the rate of variation of 241 Red Fuji in different regions was rarely low.

242

243 Phylogenetic analysis

244 Phylogenetic analysis using Maximum likelihood (ML) yielded well-supported tree topologies 245 (Fig. 6). All red Fuji are clustered into one branch, M. sieversii and M. sylvestris, also clustered 
246 into a branch, that diverged earlier than Red Fuji, suggesting that they are the ancestors of the

247 cultivated species.

248

\section{Discussion}

250 This study compared the cp genome sequences of 24 Red Fuji apples The genomes were very

251 similar to those of other Malus species regarding genome size, gene content, gene sequence, and

252 GC content (Bao et al., 2016). The length of all the cp genomes was very similar, ranging from

253160,063 to $160,070 \mathrm{bp}, \mathrm{t}$ The length of the cp genomes of $M$. sieversii is $160,025 \mathrm{bp}$, and that of

254 M. sylvestris 160,068 bp. These are slightly different from that of Red Fuji. All the genomes

255 encoded 131 genes, which were predicted to be protein-coding genes (84), tRNA genes (37), and

256 rRNA genes (8). The GC content was highly conserved (36.6\%). However, there was significant

257 diversity in the GC content among the different regions of the cp genome. The GC content in the

258 IR region was significantly higher than that in other regions, which may be due to the

259 distribution of rRNA genes.

260 Moreover, the GC content of the rRNA gene is more abundant. Previous studies have shown that

261 the IR region plays a significant role in preserving essential genes and stabilizing cp DNA

262 structure (Palmer \& Thompson, 1982; Asaf et al., 2018). In general, the change of cp genome length

263 is due to the contraction and expansion of the IR region, which is also a common evolutionary

264 phenomenon in plants (Wang et al., 2008). In our study, the length of the chloroplast genome of

265 Red Fuji in different regions was conserved (26,307-26,308 bp). The gene distribution in the

266 border regions was similar, with no apparent changes. Gene deletion and gene rearrangement

267 often occur in the genome of terrestrial plants (Knox \& Palmer, 1998). However the cp genome of

268 Red Fuji is highly conserved, and its characteristics are consistent with the slow evolution of the

269 cp genome of terrestrial plants (Rivas, 2002).

270 As the linker of nucleic acids and proteins, codons play a significant role in genetic

271 information transmission (Zhang et al., 2019). Codon use biased genetic information carrier

272 molecular DNA translation is related to the synthesis of biological functional molecular proteins,

273 which is of great significance (Quaxet al., 2015). However, in the $24 \mathrm{cp}$ Red Fuji genomes

274 sequenced, there was no difference in codon usage, and the most used amino acids were leucine,

275 serine, and arginine. Codons containing $\mathrm{A}$ and $\mathrm{T}$ bases, in which the third ended with $\mathrm{A}$ and $\mathrm{T}$,

276 were preferred. This result is consistent with the previous analysis of codon preference in the $\mathrm{cp}$

277 genome in the Rosaceae (Liu et al., 2021). 
278 Previous research has suggested that repetitive sequences significantly influence the sequence 279 differences of the cp genome (Bausher et al., 2006). We identified 1,176 repeats, each of which 280 had 49 repeats, the most common of which were positive repeat sequences. Each material 281 detected a consistent number of repeats, which showed almost no diversity between them. 282 Chloroplast SSR loci are often used to identify species and heritage analysis because of their 283 abundant distribution and high polymorphism (Wenpan et al., 2012; Morgante et al., 2013). A 284 total of 1,380 SSR sequences were detected, which were mononucleotide repeats. Most of the 285 cpSSRs were short polyadenine (poly-A) or polythymine (poly-T) repeats. Thus, cpSSR markers 286 developed in the Red Fuji cp genome could evaluate genetic diversity and potentially to 287 distinguish different germplasm (Yi et al., 2012). This study, showed that the repetitive sequences 288 and SSR loci from the nine regions have high similarity, which is also evidence of the low rate of 289 cp genome variation in Red Fuji. The whole sequence alignment of cp genomes between $M$. 290 sieversii and Red Fuji from the nine different regions showed high sequence identity with species 291 in the Rosaceae. Phylogenetic analysis showed the same results. The coding regions were more 292 conserved than the non-coding regions, and the LSC and SSC regions were more divergent from 293 each other than the IR regions. Mutations in the inverted region, are transformed, reducing this 294 region's mutation frequency (Shaw \& Small, 2005). The nucleotide polymorphism of the 24 295 materials was 0, and no SNPs were detected. There were only two indel sites in the rps 16 and $296 n d h$ A genes. The above results further confirm that the variation rate of cp DNA of Red Fuji 297 apples in different regions was very low. A low mutation rate was also found in Namrata Sarkar 298 (2017) at the somatic level. Other studies on the cp genome level also show high conservation of 299 the cp genome. For example, Feng et al. (2020) explored the cp genome structure variation of 300 wild and cultivated Qak, and found that gene number and gene structure were almost identical, 301 demonstrating that the cp genome is conserved, but cultivated species have more variation sites 302 than wild species. Muraguri et al. (2020) also found that the structure and content of the cp 303 genome in castor are conservative.

304

\section{Conclusions}

306 In the present research, the complete cp genome of Red Fuji was assembled de novo using 307 Illumina high-throughput sequencing data. The genomic structures of the 24 samples were 308 compared and analyzed. On this basis, we concluded that the cp genome structure and gene 309 content of Red Fuji apples in different regions showed little difference. Sequence alignment 
310 showed almost no variation in the chloroplast DNA sequence of Red Fuji apples in the different

311 areas. These results are consistent with previous studies reporting slow cp genome evolution.

312 These findings provide a theoretical basis and technical support for the genetic breeding of 313 apples.

314

315 References

316 Alexandra D, Tamir T. 2014. The effect of tRNA levels on decoding times of mRNA codons.

$317 \quad$ Nucleic Acids Research(14):9171 DOI: 10.1093/nar/gku646.

318 Asaf S, Khan AL, Khan MA, Shahzad R, Lubna, Kang SM, Alharrasi A, Alrawahi A, Lee IJ.

319 2018. Complete chloroplast genome sequence and comparative analysis of loblolly pine

320

(Pinus taeda L.) with related species. Plos One13(3):e0192966. DOI:

321 10.1371/journal.pone.0192966.

322

323

324

325

326

327

328

329

330

331

332

333

334

335

336

337

338

339

Abdullah, Henriquez CL, Mehmood F, Shahzadi I, Ahmed I. 2020. Comparison of Chloroplast Genomes among Species of Unisexual and Bisexual Clades of the Monocot Family Araceae. Plants9(6):737. DOI: 10.3390/plants9060737.

Alzahrani DA. 2021. Complete Chloroplast Genome of Abutilon fruticosum: Genome Structure, Comparative and Phylogenetic Analysis. Plants10(2):270. DOI: 10.3390/plants10020270.

Bausher MG, Singh ND, Lee SB, Jansen RK, Daniell H. 2006. The complete chloroplast genome sequence of Citrus sinensis (L.) Osbeck var 'Ridge Pineapple': organization and phylogenetic relationships to other angiosperms. BMC Plant Biology6(1):21. DOI: 10.1186/1471-2229-6-21.

Bao L, Li K, Liu Z, Han M, Zhang D. 2016. Characterization of the complete chloroplast genome of the Chinese crabapple Malus prunifolia (Rosales: Rosaceae: Maloideae). Conservation Genetics Resources8(3):227-229. DOI: 10.1007/s12686-016-0540-0.

Deng DJ, Li WS. 2020. The present situation and development countermeasure of Apple production and processing in China. Modern Food21:12-14. DOI: 10.16736/j.cnki.cn411434/ts.2020.21.002.

Frazer KA, Choudhuri JV, Enno O, Chris S, Jens S, Robert G. 2001. Reputer: the manifold applications of repeat analysis on a genomic scale. Nucleic Acids Research29(22):46334642. DOI: 10.1093/nar/29.22.4633. 
340 Frazer K, Lior P, Alexander P, Rubin EM, Inna D. 2004. Vista: computational tools for

341

342

343

344

345

346

347

348

349

350

351

352

353

354

355

356

357

358

359

360

361

362

363

364

365

366

367

368

369

370

371 comparative genomics. Nucleic Acids Research32(Web Server issue):W273 DOI: 10.1093/nar/gkh458.

Fu H, Yuan G, Zhong J, Cao T, Ni L, Xie P, Clair S. 2013. Environmental and Ontogenetic Effects on Intraspecific Trait Variation of a Macrophyte Species across Five Ecological Scales. Plos One8(4):e62794. DOI: 10.1371/journal.pone.0062794.

Feng LY, Liu J, Gao CW, Wu HB, Gao LZ. 2020. Higher Genomic Variation in Wild Than Cultivated Rubber Trees, Hevea brasiliensis, Revealed by Comparative Analyses of Chloroplast Genomes. Frontiers in Ecology and Evolution8:237. DOI: 10.3389/fevo.2020.00237.

Guo YY, Yang JX, Li HK, Zhao HS. 2021. Chloroplast Genomes of Two Species of Cypripedium: Expanded Genome Size and Proliferation of AT-Biased Repeat Sequences. Frontiers in Plant Science9(12):609729. DOI: 10.3389/FPLS.2021.609729.

Hummer KE, Janick J. 2009. Rosaceae: Taxonomy, Economic Importance, Genomics. Springer New York6(1):1-17. DOI:10.1007/978-0-387-77491-6_1.

He XE, Zhang CG. 2020. Analysis on Cultivation and management techniques of Fuji apple. South China Agriculture14(15):2. DOI: 10.19415/j.cnki.1673-890x.2020.15.016.

Jansen, R. K. 2007. Analysis of 81 genes from 64 plastid genomes resolves relationships in angiosperms and identifies genome-scale evolutionary patterns. Proceedings of the National Academy of Sciences 104(49):19369-19374. DOI: 10.1073/pnas.0709121104.

Jiao Y, Zhao H, Ren L, Song W, Zeng B, Guo J, Wang B, Liu Z, Chen J, Li W. 2014. Genomewide genetic changes during modern breeding of maize. Nature Genetics46(9):1039-1040. DOI: $10.1038 / \mathrm{ng} 0914-1039$.

Jung S, D Main. 2014. Genomics and bioinformatics resources for translational science in Rosaceae. Plant Biotechnology Reports8(2):49-64. DOI: 10.1007/s11816-013-0282-3.

KNOX, EB, PALMER JD. 1998. Chloroplast DNA evidence on the origin and radiation of the giant lobenas in eastern Africa. Systematic Botany23(2):109-149.

Kuang LX, Nie JY, Li YP, Cheng Y, Shen YM. 2020. Quality Evaluation of 'Fuji' Apples Cultivated in Different Regions of China. Scientia Agricultura Sinica53:2253-2263. DOI: 10.3864/j.issn.0578-1752.2020.11.011.

Liu C, Shi L, Zhu Y, Chen H, Zhang J, Lin X, Guan X. 2012. CpGAVAS, an integrated web server for the annotation, visualization, analysis, and GenBank submission of completely 
372

373

374

375

376

377

378

379

380

381

382

383

384

385

386

387

388

389

390

391

392

393

394

395

396

397

398

399

400

401

402

403

sequenced chloroplast genome sequences. BMC Genomics13(1):715-715. DOI: 10.1186/1471-2164-13-715.

Li Y, Zhang J, Li L, Gao L, Xu J, Yang M. 2018. Structural and Comparative Analysis of the Complete Chloroplast Genome of Pyrus hopeiensis - "Wild Plants with a Tiny Population"-and Three Other Pyrus Species. International Journal of Molecular ences Sciences 19(10):3262. DOI: 10.3390/ijms19103262.

Li M, Guo J, He J, Xu C, Tao S. 2019. Possible impact of climate change on apple yield in Northwest China. Theoretical and Applied Climatology(3). DOI: 10.1007/s00704-01902965-y.

Li Q, Sun XR, Wang J. 2019. Discussion on growth characteristics and Cultivation Techniques of Red Fuji apple trees. Nong Jia Can Mou615(07):94. DOI: CNKI:SUN:NJCM.0.2019-07080.

Yan M, Zhao X, Zhou J, Huo Y, Yuan Z. 2019. The complete chloroplast genome of cultivated apple (Malus domestica cv. 'yantai Fuji 8'). Mitochondrial DNA Part B4(1):1213-1216. DOI: 10.1080/23802359.2019.1591182.

Liu XY, He ZJ, Qiu YM. 2021. Codon Bias in the Chloroplast Genome of Four Rosaceae Fruit Trees. Molecular Plant Breeding1-13.

Morgante M, Hanafey M, Powell W. 2002. Microsatellites are preferentially associated with nonrepetitive DNA in plant genomes. Nature Genetics30(2):194-200. DOI: 10.1063/1.363554.

Moore MJ, Soltis PS, Bell CD, Burleigh JG, Soltis DE. 2010. Phylogenetic analysis of 83 plastid genes further resolves the early diversification of eudicots. Proceedings of the National Academy of Sciences of the United States of America 107(10):4623-4628. DOI: 10.1073/pnas.0907801107.

Muraguri S, Xu W, Chapman M, Muchugi A, Li A. 2020. Intraspecific variation within Castor bean (Ricinus communis L.) based on chloroplast genomes. Industrial Crops and Products 155:112779. DOI: 10.1016/j.indcrop.2020.112779.

Namrata S, Emanuel S, Christian I, Sandra C, Caroline GD, Jacqueline C, Pietro C, Frédéric Schütz, Laurent F, Marco P, Michel S, Jérémie V, Michel J, Christian F, Christian SH, Laurent K, John RP, Alexandre R, Marc RR, Ioannis X, Philippe R. 2017. Low rate of somatic mutations in a long-lived oak tree. BioRxiv3:926-929. DOI: http://dx.doi.org/10.1101/149203. 
404 Na An, Sheng F, Yang Y, Xilong C, Feng D, Yibin W, Libo X, Caiping Z, Mingyu H. 2018.

405 Identification and Characterization of miRNAs in Self-Rooted and Grafted Malus Reveals

406 Critical Networks Associated with Flowering. International Journal of Molecular

407 Sciences19(8):2384. DOI: 10.3390/ijms19082384.

408 Ohyama K, Fukuzawa H, Kohchi T, Shirai H, SanoT, Sano S, Kazuhiko U, Yasuhiko S,

409 Masayuki T, Zhen C. 1986. Chloroplast gene organization deduced from complete sequence

410 of liverwort Marchantia polymorpha chloroplast DNA. Nature 322(6079):572-574. DOI:

$411 \quad 10.1038 / 322572 \mathrm{a} 0$.

412 Palmer JD, Thompson WF. 1982. Chloroplast DNA rearrangements are more frequent when a

413 large inverted repeat sequence is lost. Cell29(2):537-550. DOI: 10.1016/0092-

414 8674(82)90170-2.

415 Qian J, Song J, Gao H, Zhu Y, Xu J, Pang X, 2013. The complete chloroplast genome sequence

416 of the medicinal plant Salvia miltiorrhiza. Plos One8(2): e57607. DOI:

$417 \quad$ 10.1371/journal.pone.0057607

418 Quax TF, Claassens N, SL1 D, VanDerOost J. 2015. Codon Bias as a Means to Fine-Tune Gene

419 Expression. Molecular cell59(2):149-161. DOI: 10.1016/j.molcel.2015.05.035.

420 Rochaix, D. 1978. Restriction endonuclease map of the chloroplast DNA of Chlamydomonas

421 reinhardii. Journal of Molecular Biology126(4):597-617. DOI: 10.1016/0022-

$422 \quad 2836(78) 90011-6$.

423 Rivas, DI J. 2002. Comparative Analysis of Chloroplast Genomes: Functional Annotation,

424 Genome-Based Phylogeny, and Deduced Evolutionary Patterns. Genome

425 Research12(4):567-583. DOI: 10.1101/gr.209402.

426 Raubeson LA, Peery R, Chumley TW, Dziubek C, Fourcade HM, Boore JL, Jansen RK. 2007.

427 Comparative chloroplast genomics: analyses including new sequences from the

428 angiosperms Nupharadvenaand Ranunculus macranthus. BMC Genomics8(1):174. DOI:

$429 \quad 10.1186 / 1471-2164-8-174$.

430 Shinozaki K, Ohme M, Tanaka M, Wakasugi T, Sugiura M.1986. The complete nucleotide

431

432

sequence of the tobacco chloroplast genome: its gene organization and expression. Plant

433 Sugiura, M. 2003. History of chloroplast genomics. Photosynthesis. Research 76(1-3):371-377.

434 DOI: $10.1023 / \mathrm{A}: 1024913304263$. 
435 Shaw J, Small RL. 2005. Chloroplast DNA phylogeny and phylogeography of the North

436

437

438

439

440

441

442

443

444

445

446

447

448

449

450

451

452

453

454

455

456

457

458

459

460

461

462 American plums (Prunus subgenus Prunus section Prunocerasus, Rosaceae). American Journal of Botany92(12):2011-2030. DOI: 10.3732/ajb.92.12.2011.

Salichos Stamatakis, Rokas. 2014. Novel information theory-based measures for quantifying incongruence among phylogenetic trees. MOL BIOL EVOL31(5):1261-1271. DOI: 10.1093/molbev/msu061.

Thiel T, Michalek W, Varshney R, Graner A. 2003. Exploiting est databases for the development and characterization of gene-derived ssr-markers in barley (hordeum vulgare L.). Theoretical \& Applied Genetics106(3):411-422. DOI: 10.1007/s00122-002-1031-0.

Wang RJ, Cheng CL, Chang CC, Wu CL, Su TM, Chaw SM. 2008. Dynamics and evolution of the inverted repeat-large single copy junctions in the chloroplast genomes of monocots. BMC Evolutionary Biology8(1):36. DOI: 10.1186/1471-2148-8-36.

Wenpan D, Xu C, Cheng T, Lin K, Zhou S. 2013. Sequencing Angiosperm Plastid Genomes Made Easy: A Complete Set of Universal Primers and a Case Study on the Phylogeny of Saxifragales. Genome Biology \& Evolution5(5):989-997. DOI: 10.1093/gbe/evt063.

Wang J, Mu W, Yang T, Song Y, Zhao H. 2021. Targeted Enrichment of Novel ChloroplastBased Probes Reveals a Large-Scale Phylogeny of 412 Bamboos. BMC Plant Biology21(1):76. DOI: 10.1186/s12870-020-02779-5.

Yi DK, Kim KJ, Zhang J. 2012. Complete Chloroplast Genome Sequences of Important Oilseed Crop Sesamum indicum L. Plos One7(5):e35872. DOI: 10.1371/journal.pone.0035872.

Yagi Y, Shiina T. 2014. Recent advances in the study of chloroplast gene expression and its evolution. Frontiers in Plant ence5(1):61. DOI: 10.3389/fpls.2014.00061.

Zuo LH, Shang AQ, Zhang S, Yu XY, Wang JM. 2017. The first complete chloroplast genome sequences of Ulmus species by de novo sequencing: Genome comparative and taxonomic position analysis. Plos One12(2):e0171264. DOI: 10.1371/journal.pone.0171264.

Zhang J, Jiang Z, Su H, Zhao H, Cai J. 2019. The complete chloroplast genome sequence of the endangered species Syringa pinnatifolia (Oleaceae). Nordic Journal of Botany1-11. DOI: 10.1111/njb.02201. 
Figure 1

Genome map of Red Fuji chloroplast.

Different colors represent different genes. The inner genesare transcribed clockwise, the outer genesare transcribed counterclockwise. The innermost gray pattern represents GC content 


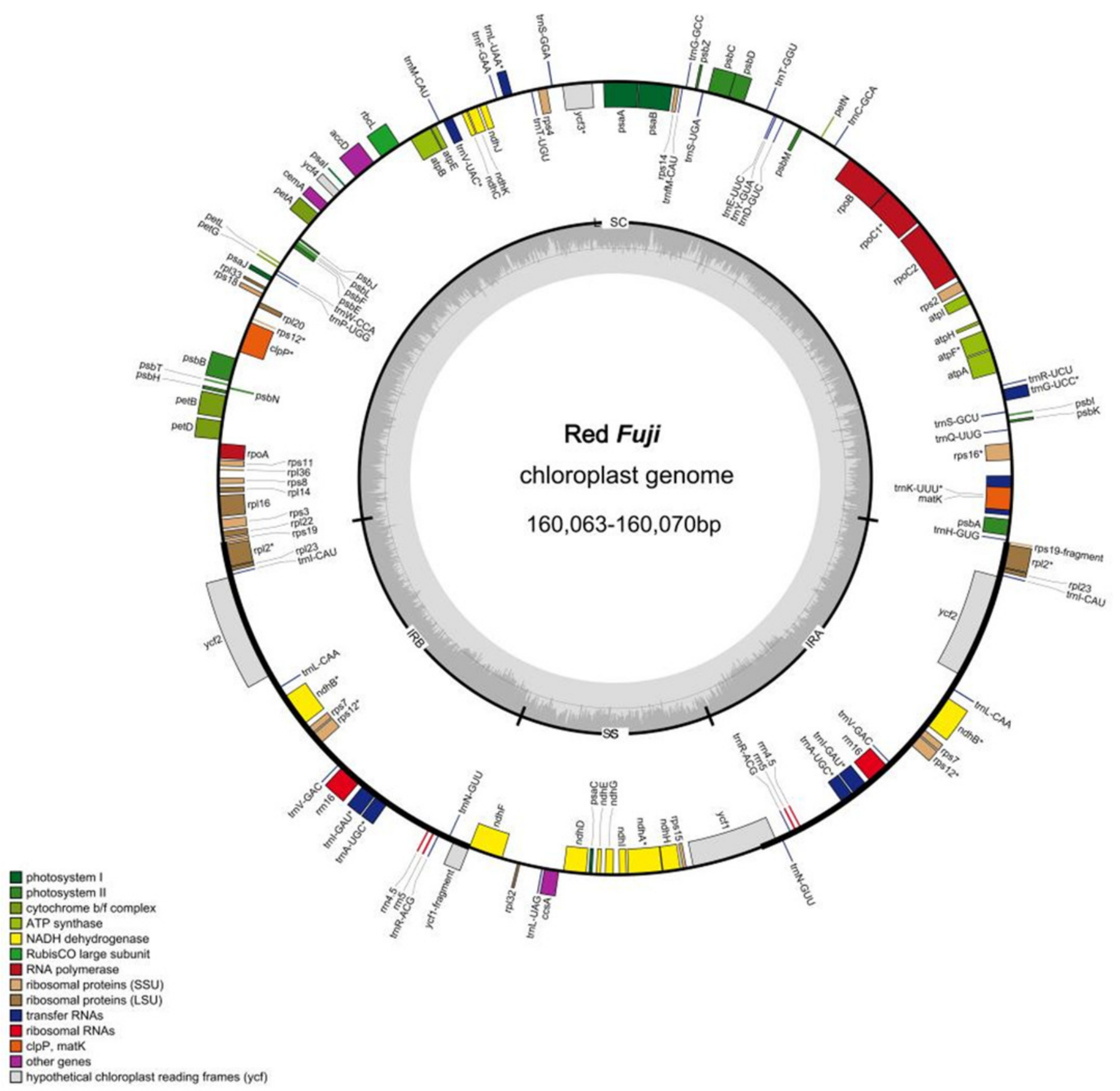


Figure 2

Comparison of chloroplast genome boundary regions in Red Fuji, M. sieversii and $M$. sylvestris

\section{Inverted Repeats}

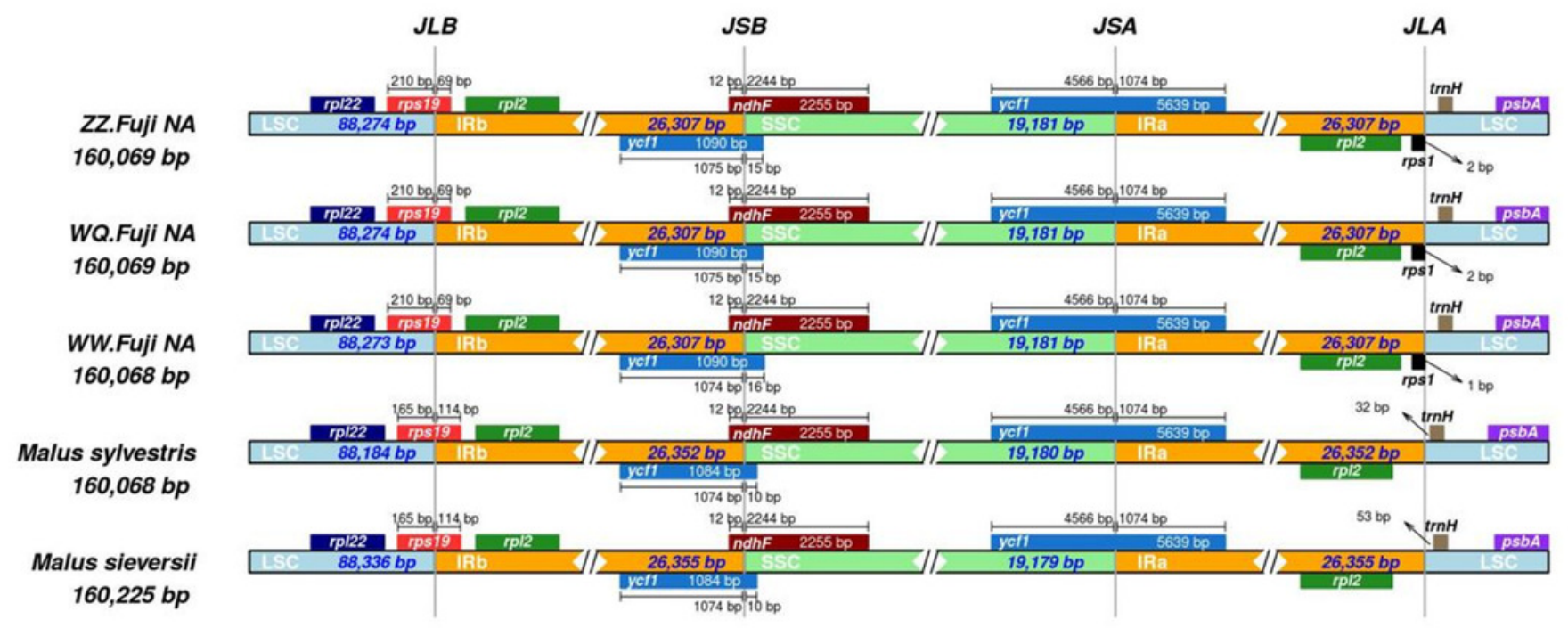




\section{Figure 3}

Codon content of amino acids encoding proteins in the chloroplast genome of Red Fuji

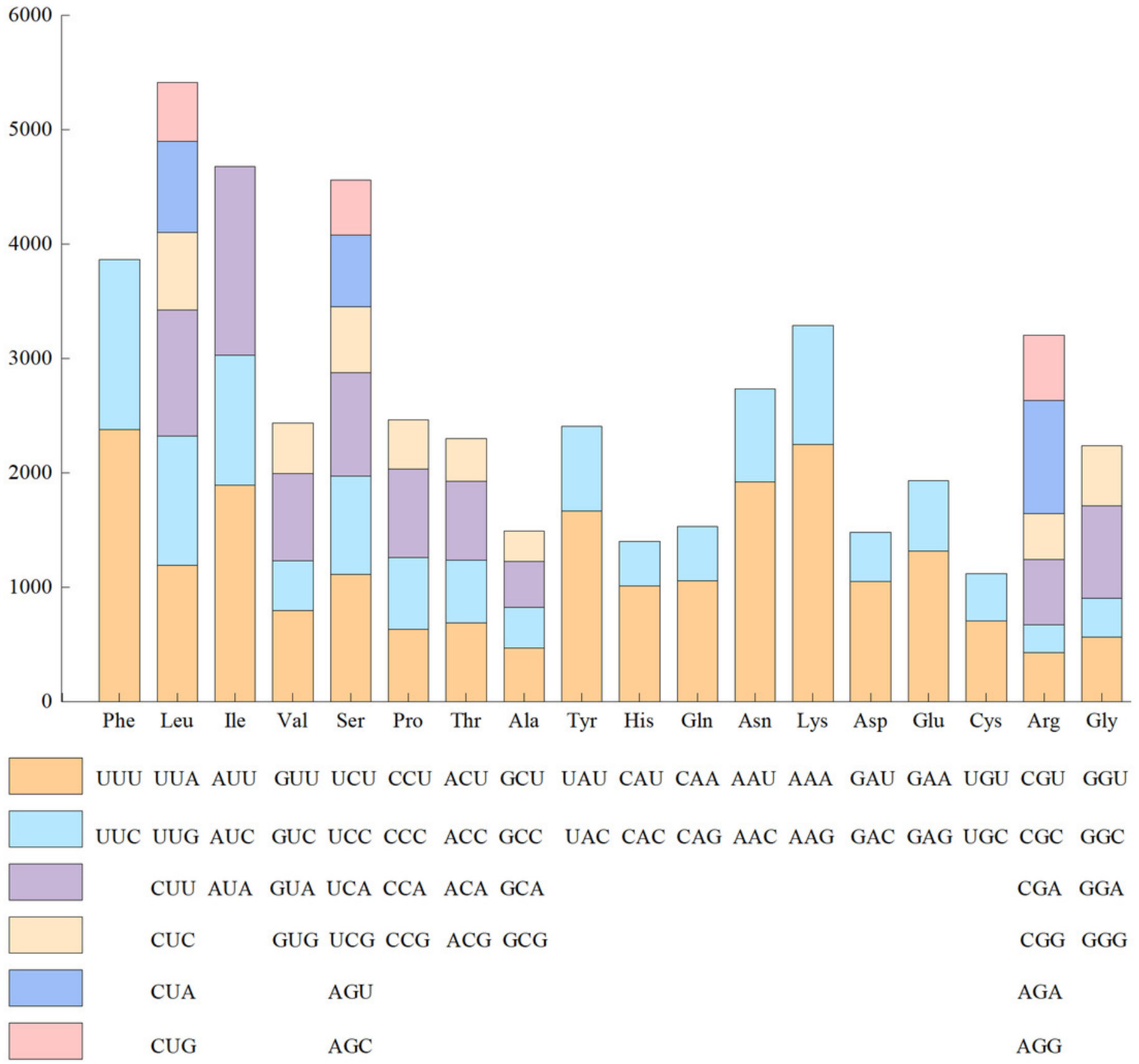




\section{Figure 4}

Length and number of different types of long repeats.

C: Complementary-type repetition. F: Forward repetition. P: Palindrome repetition. R: Reverse repetition
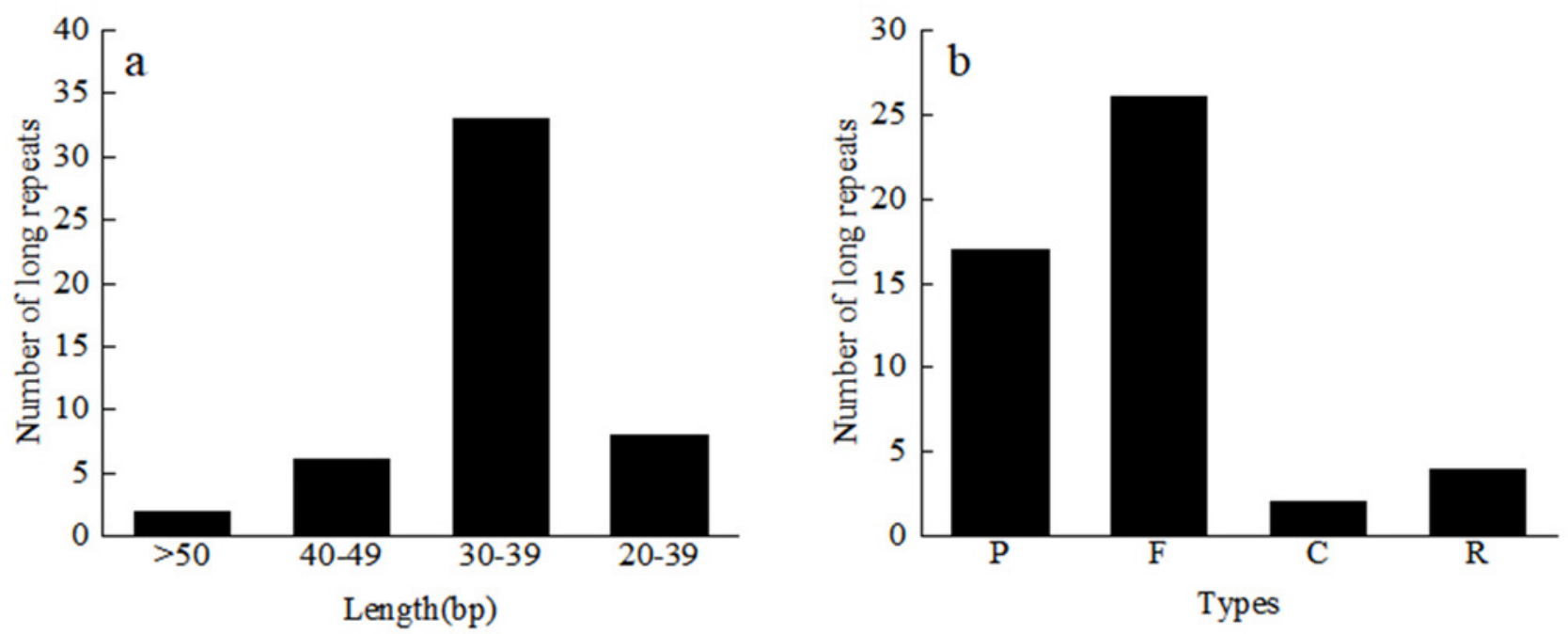


\section{Figure 5}

Sequence alignment of nine Red Fuji chloroplast genomes using the mVISTA software with $M$. sieversii as a reference.

The $y$-axis represents the percent identity within $50-100 \%$. The transcriptional direction of genes is indicated by grey arrows. Genome regions are color-coded as protein-coding (exon), tRNA, rRNA, and conserved non-coding sequences (CNS)

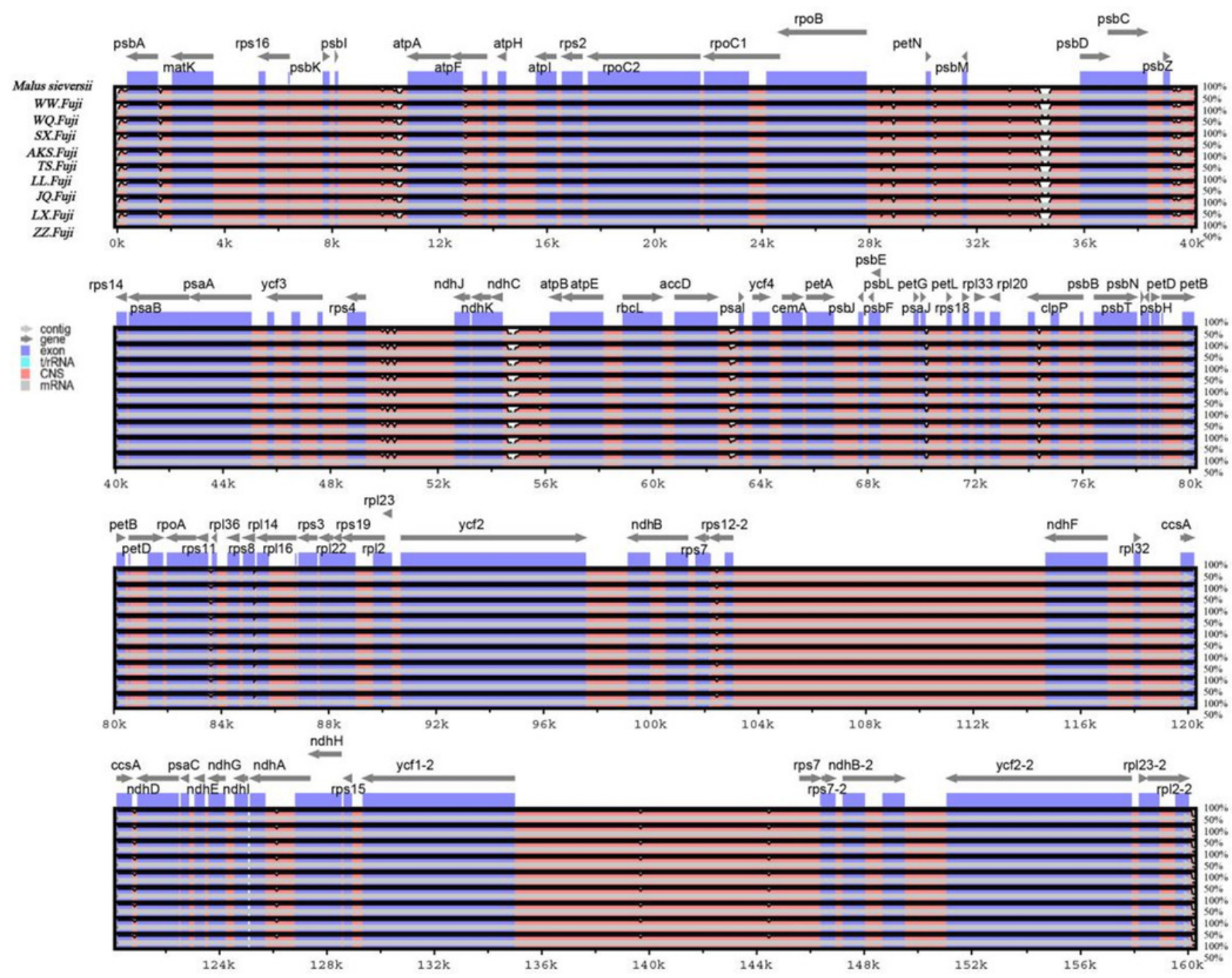


Figure 6

Phylogenetic tree reconstruction using maximum likelihood.

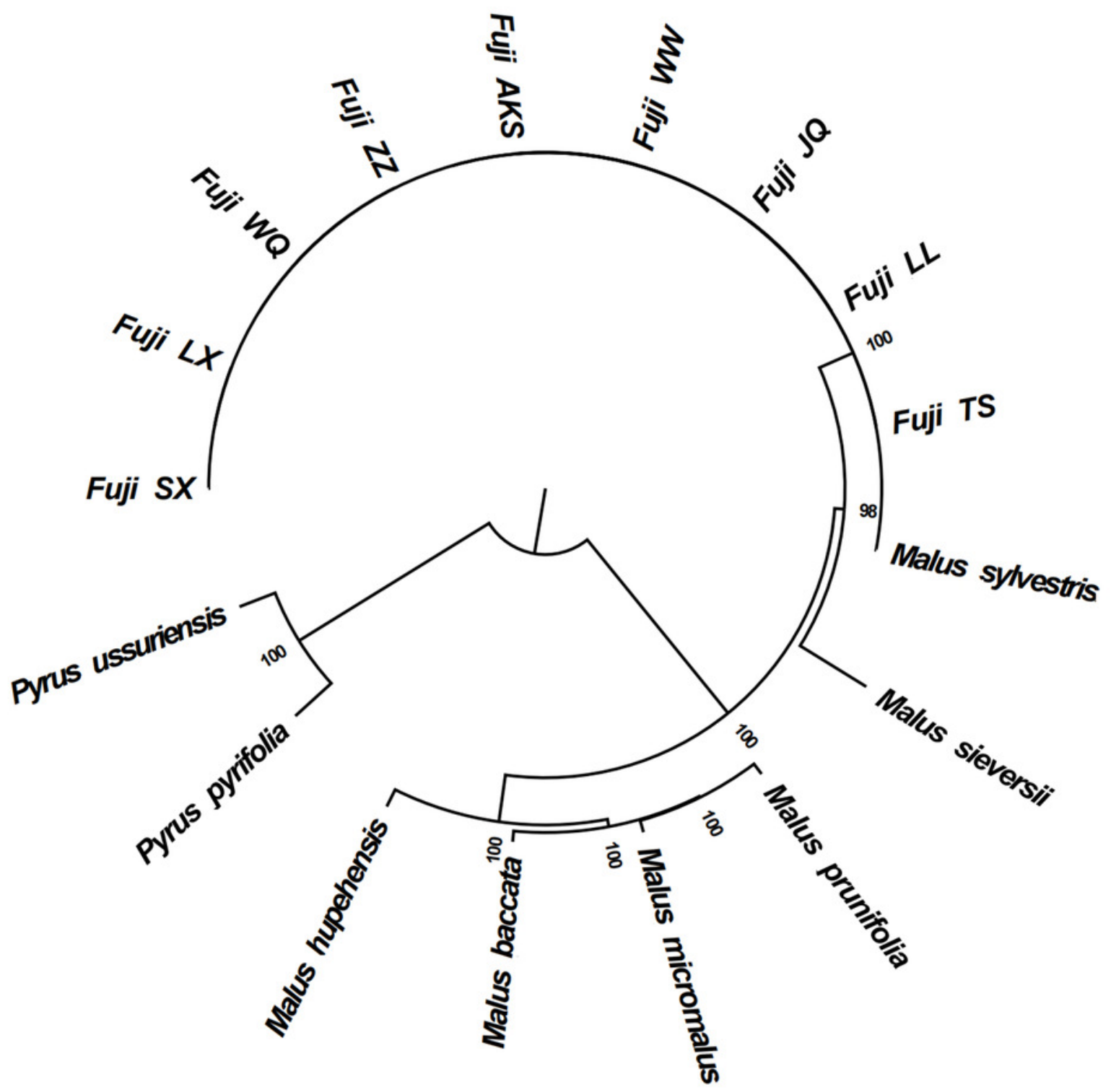




\section{Table $\mathbf{1}$ (on next page)}

Germplasm resources of Red Fuji apple in nine regions. 


\begin{tabular}{cccc}
\hline & Region & Amount & GenBank numbers \\
\hline 1 & Wunan Town, Wuwei City & 2 & OK458680, OK585094 \\
2 & Qingyuan Town, Wuwei City & 2 & OK514184, OK585095 \\
3 & Shanxi Province & 3 & OK585096, OK514185, OK585097 \\
4 & Aksu & 3 & OK514186, OK585098, OK585099 \\
5 & Tianshui City & 3 & OK585100, OK514187, OK585101 \\
6 & Li County, Longnan & 3 & OK514188, OK585102, OK585103 \\
7 & Jiuquan city & 3 & OK514189, OK585104, OK585105 \\
8 & Xingcheng City, Liaoning Province & 2 & OK514190, OK585106 \\
9 & Zhengzhou Fruit Tree Institute & 3 & OK585107, OK514191, OK585108 \\
\hline
\end{tabular}


Table 2 (on next page)

Statistics of the chloroplast genomes of Red Fuji. 


\begin{tabular}{ccccccccc}
\hline & $\begin{array}{c}\text { Size } \\
(\mathrm{bp})\end{array}$ & $\begin{array}{c}\text { LSC } \\
(\mathrm{bp})\end{array}$ & $\begin{array}{c}\text { SSC } \\
(\mathrm{bp})\end{array}$ & $\begin{array}{c}\text { IR } \\
(\mathrm{bp})\end{array}$ & $\begin{array}{c}\text { protein-coding } \\
\text { genes }\end{array}$ & $\begin{array}{c}\text { tRNA } \\
\text { genes }\end{array}$ & $\begin{array}{c}\text { rRNA } \\
\text { genes }\end{array}$ & $\begin{array}{c}\text { GC } \\
\text { content(\%) }\end{array}$ \\
\hline WW & 160068 & 88273 & 19181 & 26307 & 86 & 37 & 8 & 36.6 \\
WQ & 160069 & 88274 & 19181 & 26307 & 86 & 37 & 8 & 36.6 \\
SX & 160069 & 88274 & 19181 & 26307 & 86 & 37 & 8 & 36.6 \\
AKS & 160068 & 88273 & 19181 & 26307 & 86 & 37 & 8 & 36.6 \\
ZZ & 160070 & 88274 & 19181 & 26308 & 86 & 37 & 8 & 36.6 \\
TS & 160069 & 88274 & 19181 & 26307 & 86 & 37 & 8 & 36.6 \\
LL & 160066 & 88272 & 19180 & 26307 & 86 & 37 & 8 & 36.6 \\
LX & 160063 & 88273 & 19176 & 26307 & 86 & 37 & 8 & 36.6 \\
JQ & 160067 & 88272 & 19181 & 26907 & 86 & 37 & 8 & 36.6 \\
\hline
\end{tabular}

WW: Wunan Town, Wuwei City. WQ: Qingyuan Town, Wuwei City. SX: Shanxi Province . AKS: Aksu. ZZ: Zhengzhou Fruit Tree Institute. TS:TianshuiCity. LL: Li County, Longnan. JQ: jiuquan city. LX: Xingcheng City, Liaoning Province 


\section{Table 3 (on next page)}

Base composition of chloroplast genome of Red Fuji apple in different regions. 


\begin{tabular}{|c|c|c|c|c|c|c|c|c|c|c|}
\hline & & WW & WQ & SX & AKS & $\mathrm{ZZ}$ & $\mathrm{TS}$ & LL & LX & JQ \\
\hline \multirow[t]{5}{*}{$\operatorname{LSC}(\%)$} & A & 32.2 & 32.2 & 32.2 & 32.2 & 32.2 & 32.2 & 32.2 & 32.2 & 32.2 \\
\hline & $\mathrm{C}$ & 17.6 & 17.6 & 17.6 & 17.6 & 17.6 & 17.6 & 17.6 & 17.6 & 17.6 \\
\hline & G & 16.6 & 16.6 & 16.6 & 16.6 & 16.6 & 16.6 & 16.6 & 16.6 & 16.6 \\
\hline & $\mathrm{T}$ & 33.6 & 33.6 & 33.6 & 33.6 & 33.6 & 33.6 & 33.6 & 33.6 & 33.6 \\
\hline & GC & 34.2 & 34.2 & 34.2 & 34.2 & 34.2 & 34.2 & 34.2 & 34.2 & 34.2 \\
\hline \multirow[t]{5}{*}{$\mathrm{SSC}(\%)$} & A & 34.6 & 34.7 & 34.7 & 34.7 & 34.7 & 34.7 & 34.7 & 34.7 & 34.7 \\
\hline & $\mathrm{C}$ & 15.9 & 15.9 & 15.9 & 15.9 & 15.9 & 15.9 & 15.9 & 15.9 & 15.9 \\
\hline & G & 14.5 & 14.5 & 14.5 & 14.5 & 14.5 & 14.5 & 14.5 & 14.5 & 14.5 \\
\hline & $\mathrm{T}$ & 34.8 & 34.8 & 34.8 & 34.8 & 34.8 & 34.8 & 34.8 & 34.8 & 34.8 \\
\hline & GC & 30.4 & 30.4 & 30.4 & 30.4 & 30.4 & 30.4 & 30.4 & 30.4 & 30.4 \\
\hline \multirow[t]{5}{*}{$\operatorname{IRa}(\%)$} & A & 28.5 & 28.5 & 28.5 & 28.5 & 28.5 & 28.5 & 28.5 & 28.5 & 28.5 \\
\hline & $\mathrm{C}$ & 22.1 & 22.1 & 22.1 & 22.1 & 22.1 & 22.1 & 22.1 & 22.1 & 22.1 \\
\hline & G & 20.6 & 20.6 & 20.6 & 20.6 & 20.6 & 20.6 & 20.6 & 20.6 & 20.6 \\
\hline & $\mathrm{T}$ & 28.8 & 28.8 & 28.8 & 28.8 & 28.8 & 28.8 & 28.8 & 28.8 & 28.8 \\
\hline & GC & 42.7 & 42.7 & 42.7 & 42.7 & 42.7 & 42.7 & 42.7 & 42.7 & 42.7 \\
\hline \multirow[t]{5}{*}{ Total(\%) } & A & 24.3 & 24.3 & 24.3 & 24.3 & 24.3 & 24.3 & 24.3 & 24.3 & 24.3 \\
\hline & $\mathrm{C}$ & 18.6 & 18.6 & 18.6 & 18.6 & 18.6 & 18.6 & 18.6 & 18.6 & 18.6 \\
\hline & G & 17.9 & 17.9 & 17.9 & 17.9 & 17.9 & 17.9 & 17.9 & 17.9 & 17.9 \\
\hline & $\mathrm{T}$ & 32.1 & 32.1 & 32.1 & 32.1 & 32.1 & 32.1 & 32.1 & 32.1 & 32.1 \\
\hline & GC & 36.6 & 36.6 & 36.6 & 36.6 & 36.6 & 36.6 & 36.6 & 36.6 & 36.6 \\
\hline
\end{tabular}




\section{Table 4 (on next page)}

Statistics of simple repeats (SSRs) of chloroplast genome. 


\begin{tabular}{llllllllll}
\hline & WW & WQ & SX & AKS & TS & LL & JQ & LX & ZZ \\
\hline SSR & 57 & 58 & 58 & 57 & 58 & 57 & 57 & 57 & 58 \\
P1 & 46 & 48 & 47 & 47 & 47 & 47 & 46 & 46 & 47 \\
P2 & 2 & 2 & 2 & 2 & 2 & 2 & 2 & 2 & 2 \\
Pc & 9 & 9 & 9 & 9 & 9 & 8 & 9 & 9 & 9 \\
LSC & 46 & 47 & 47 & 46 & 47 & 46 & 46 & 46 & 47 \\
SSC & 5 & 5 & 5 & 5 & 5 & 5 & 5 & 5 & 5 \\
IR & 6 & 6 & 6 & 6 & 6 & 6 & 6 & 6 & 6 \\
\hline P1. mononucleotide. P2.
\end{tabular}

